\title{
Linx
}

Revue des linguistes de l'université Paris X Nanterre

70-71| 2014

Variations sémantiques et syntaxiques : aspects d'une théorie de l'invariance

\section{Effets sémantiques, syntaxiques et énonciatifs du jeu entre quantité et qualité}

\section{Sarah de Vogüé}

\section{(2) OpenEdition \\ Journals}

Édition électronique

URL : http://journals.openedition.org/linx/1575

DOI : $10.4000 /$ linx.1575

ISSN : 2118-9692

Éditeur

Presses universitaires de Paris Nanterre

Édition imprimée

Date de publication : 1 septembre 2014

Pagination : 141-164

ISSN : 0246-8743

Référence électronique

Sarah de Vogüé, "Effets sémantiques, syntaxiques et énonciatifs du jeu entre quantité et qualité ", Linx [En ligne], 70-71 | 2014, mis en ligne le 01 mars 2015, consulté le 06 mai 2019. URL : http:// journals.openedition.org/linx/1575; DOI : 10.4000/linx.1575 


\section{Effets sémantiques, syntaxiques et énonciatifs du jeu entre quantité et qualité}

Sarah de Vogüé, Université Paris Ouest Nanterre la Défense, MoDyCo CNRS

\section{Introduction}

On se propose ici de mettre en évidence un principe général de variation dont on soutiendra qu'il est à l'œuvre dans tous les plans de structuration du sens, depuis le lexique jusqu'au discours en passant par la syntaxe et la morphologie : car il touche à une question qui traverse tous ces plans, la question de la référence. Ce principe concerne le jeu qui s'instaure entre ce que l'on appellera une référence quantitative et 
une référence qualitative ${ }^{1}$ : on soutiendra en effet qu'il y a plusieurs façons de construire la référence, un mode quantitatif et un mode qualitatif, qui peuvent en outre s'articuler. D'où un jeu de bascule qui engendre cette variation régulière.

Les faits que l'on va analyser sont souvent bien connus, mais ils sont traités de manière séparée : on montrera qu'ils s'inscrivent dans un motif de variation qui est général. Aller ainsi du lexique au discours au cours d'un article impose de parcourir des exemples variés mais exemplaires, dont l'analyse ne pourra pas être approfondie, dans la mesure où il s'agira seulement de mettre en évidence le principe de variation qui nous intéresse ici : toute la démonstration repose sur le parcours effectué parmi des faits a priori différents ; elle impose par conséquent un survol, sinon des problèmes traités, du moins des problèmes que pourrait poser chaque fait pris séparément. Le bénéfice cependant de ce parcours est qu'il permet de renouveler comme on le verra à la fois la conception que l'on peut avoir des faits de polysémie lexicale (en s'opposant notamment aux théories de la métaphore qui organisent la variation autour d'un sens premier concret), mais aussi la perception que l'on a des variations énonciatives (on s'opposera ici aux théories qui réduisent l'énonciation à des degrés variés de repérage par rapport à la situation d'énonciation), et celle que l'on peut avoir de la syntaxe des propositions (on plaidera pour des principes de structuration syntaxique qui sont concurrents et non pas complémentaires, entre structure bipartite, tripartite et pluripartite à la façon des structures macro-syntaxiques, leur concurrence étant ce qui permet variation et déformation).

\section{Référence quantitative et référence qualitative : à propos de quelques grandes catégories de référence}

On propose ici une caractérisation des concepts de quantité et de qualité fondée sur les opérations linguistiques au travers desquelles cette opposition se manifeste. L'objectif est d'arriver à circonscrire les opérations linguistiques en question, et de se doter de critères propres à distinguer les catégories de référence concernées $^{2}$.

\subsection{Prédication thétique / prédication catégorique : première analyse de quelques exemples pris isolément}

On peut commencer négativement. Contrairement à divers usages courants de l'opposition entre un point de vue quantitatif et un point de vue qualitatif, la notion de quantité n'emporte pas avec elle celle de numérisation : les qualités peuvent elles aussi, au moins pour certaines d'entre elles, être graduées, et par ce biais numérisées. Une quantité ne peut donc pas se définir comme étant une entité numérique ou même seulement numérisable.

1 Les concepts de quantité et de qualité ont fait l'objet d'une première présentation ici même dans l'article de Lionel Dufaye (qui ajoute une forme d'articulation entre quantité et qualité que nous proposons ici sous une autre forme, et avec d'autres effets).

2 Voir aussi De Vogüé (1999) pour une première tentative de caractérisation sur laquelle on va s'appuyer. 
Par ailleurs il n'est pas certain que la distinction dont il est question soit repérable dans une réalité extralinguistique prédéterminée : ainsi s'il y a un sens, ainsi qu'on le montrera, à considérer au moins grossièrement que les mots eau et chand s'opposent l'un à l'autre comme étant le premier d'ordre quantitatif (parce que doté d'une référence concrète, matérielle et délimitée), le second d'ordre qualitatif (parce que propre à caractériser qualitativement des entités données indépendamment), en revanche il n'y a pas forcément grand sens à considérer que l'entité eau est plus quantitative que l'entité chaleur.

Le clivage se repère en revanche nettement dans des contrastes linguistiques variés. On citera pour une première illustration le cas de deux mots pour lesquels l'opposition entre une interprétation quantitative et une interprétation qualitative produit une variation caractéristique. C'est d'abord l'exemple du mot singulier qui peut désigner l'unique par opposition au pluriel (interprétation quantitative), ou qui peut renvoyer à une forme d'anormalité (interprétation qualitative) : on voit que la variation n'est pas sans rapport avec l'opposition entre nom et adjectif, le nom ayant plutôt la première interprétation (dans un usage en outre particulier, puisque réservé à la métalangue), tandis que l'adjectif (un comportement singulier) appelle la valeur qualitative. Cela se trouve confirmé par le second exemple, qui est celui du mot exemplaire, dont la variation est plus clairement encore liée à sa nature catégorielle, puisqu'il désigne dans une interprétation quantitative comme nominal un échantillon (un exemplaire de ce médicament), et dans une interprétation qualitative comme adjectif ce qui serait un exemple à suivre (un comportement exemplaire).

Ce contraste entre emplois nominaux et adjectivaux est révélateur de ce qui fonde effectivement l'opposition. Si l'on s'en tient en effet aux opérations linguistiques impliquées, ce qui est en jeu dans le clivage entre quantité et qualité est lié au contraste entre ce que l'on pose lorsque l'on dit que «quelque chose est» (de l'eau, un exemplaire, du chaud, un singulier), et ce que l'on pose lorsque l'on dit «comment c'est» (chaud, exemplaire, singulier). On voit que cela correspond à deux formes distinctes de prédication ${ }^{3}$ : d'un côté une prédication thétique, qui consiste à poser l'existence de quelque chose ("quelque chose est»), et dont la forme canonique est il existe $X$, ou il y a $X$; de l'autre une prédication catégorique, qui consiste à attribuer une propriété à quelque chose (en disant comment ce quelque chose est), et dont la forme canonique est binaire, $\alpha$ est $X$, avec $X$ en position de prédicat qui porte sur une autre entité, $\alpha$, introduite indépendamment, et se présentant par conséquent comme a priori extérieure à $X$.

On a donc d'un côté une prédication unaire, de l'autre une prédication binaire. La prédication thétique mobilise cependant elle aussi une forme de binarité, quoique ce soit sur un autre mode. Le fait est que la prédication d'existence emporte nécessairement avec elle une localisation, dans la mesure où la notion même d'existence suppose un site pour cette existence ${ }^{4}$ : si quelque chose est, - eau, exemplaire, chaud ou autre -, il est dès lors nécessairement situé, là où il est et pendant le temps qu'il est. Le $X$ quantité mobilise donc un site $S$, quand le $X$ qualité mobilise les arguments $\alpha$ auxquels il s'applique. Il n'en demeure pas moins que les deux prédications restent

3 Voir Kuroda (1973).

4 Ce dont le $y$ de ily a est la trace. 
fondamentalement distinctes, la relation de localisation $X$ loc $S$ n'étant pas ce que la prédication thétique pose, mais une conséquence de l'existence qu'elle pose. Le site $S$ n'a pas le statut d'un argument ; il peut rester implicite, ce qui n'aurait pas de sens pour un argument; $X$ ne s'entend pas comme étant une propriété de $S$, mais comme étant ce que $S$ situe ; et les relations de localisation et d'attribution de propriété mobilisées par les deux prédications sont orientées inversement l'une de l'autre ( $X$ loc $S / \alpha$ est $X)$.

Il est au demeurant une autre différence qui est particulièrement importante pour prendre la mesure de l'opposition entre quantités et qualités. Les qualités relevant d'un fonctionnement catégorique, les arguments auxquels une qualité $X$ s'applique se trouvent de ce fait inscrits dans une classe, la classe de toutes les entités auxquelles elle s'applique - classe de tout ce qui est chaud, exemplaire, singulier, etc. -, par opposition à la classe des entités auxquelles elle ne s'applique pas. Et c'est la qualité $X$ elle-même qui fonde le critère de délimitation entre ces deux classes.

Du côté des quantités aussi on a des affaires de délimitation, mais qui sont d'un autre ordre : étant situées dans l'espace-temps, les quantités se trouvent de ce fait même dotées d'une extension, ce qui suppose bien une délimitation de cette extension, la séparant de tout ce qui y est extérieur. Mais cette délimitation peut être strictement contingente (pourquoi y a-t-il de l'eau là et pas ailleurs ? pourquoi l'étendue d'eau s'arrête-t-elle là où elle s'arrête ?) : ce n'est pas a priori $X$ en tant que tel qui détermine cette délimitation de son extension. En outre l'extension de $X$ est donnée directement par la prédication thétique $(X e s t)$, alors que l'extension de la classe à laquelle renvoie une qualité n'est que virtuellement convoquée par la prédication catégorique, qui en attribuant à $\alpha$ la qualité $X$ l'inscrit dans la classe de tout ce qui présente cette même qualité.

Les quantités relèvent d'une prédication thétique et appellent dès lors un ancrage situationnel, quand les qualités relèvent d'une prédication catégorique et s'appliquent à des arguments pour les inscrire dans les classes qu'elles définissent. Cela suffit à nous fournir des formes de critères pour identifier les unes et les autres. On verra dans la partie suivante à déployer tout un dispositif de critères qui sont à la fois bien connus et relativement opératoires, mais on peut déjà en première approximation opposer sur la base des critères ci-dessus un verbe comme plewvoir d'un coté et un verbe comme plaire de l'autre, qui a priori renvoient plutôt le premier à des quantités, localisées (quelque chose a lieu à un moment donné et en un lieu donné, qui est de la pluie, ou plus généralement du qui pleut), et le second à une qualité, qui distingue la classe de ce qui plait de celle de ce qui ne plait pas 5 .

\subsection{Nominaux comptables et accomplissements : quand quantité et qualité se trouvent articulées}

On vient de voir que la délimitation d'une quantité peut être strictement contingente, au sens où elle n'est pas déterminée par les propriétés définitoires de la quantité en question : ainsi, rien dans la notion d'eau ne dit comment les occurrences d'eau doivent être configurées ; rien ne vient par conséquent déterminer a priori ce qui serait la délimitation de l'extension de la quantité à laquelle réfère le mot eau.

5 Que plewvoir soit un verbe impersonnel, et donc unaire, aide à l'exclure d'un fonctionnement qui serait qualitatif : il n'y a pas d'argument sur lequel il pourrait porter. 
Il est des cas cependant où le qualitatif détermine au moins partiellement l'extension du quantitatif, en définissant d'une manière ou d'une autre un format général auquel cette extension doit satisfaire. Quantité et qualité sont alors articulées : la quantité est configurée qualitativement.

Les cas en question sont bien connus. Cela correspond du côté des nominaux à ce que l'on décrit comme des nominaux comptables, qui pour être comptables doivent d'abord être formatés (de manière à ce que l'unité de compte puisse être définie). La délimitation des entités qui peuvent servir de référent à un nom comme voiture est déterminée qualitativement: elle fait partie de la notion même de voiture, qui correspond à un certain nombre de qualités mais aussi à un format.

Du côté des verbes aussi on trouve des configurations référentielles dans lesquelles le format des référents est déterminé qualitativement: ce qui vient alors opérer ainsi une découpe intrinsèque du processus auquel le verbe réfère est ce qui a été identifié depuis Aristote comme étant la fin ou finalité autour de laquelle ce processus s'organise. Il s'agit par conséquent de ce que l'on décrit comme des processus finalisés ou téliques, à savoir ces procès que Vendler classe parmi les accomplissements, dont un exemple pourrait être le procès de réparation tel que donné par le lexème du français répar- : la réparation est un processus déterminé par la finalité qu'il se donne, à savoir une réparation effective, avec comme état résultant l'objet de cette réparation qui se trouve effectivement réparé (= en état de marche). Cette finalité étant en même temps la fin du processus, on voit qu'il s'agit bien d'une délimitation de l'extension du procès, délimitation qui se trouve définie qualitativement, par un état résultant (le réparé en étant de marche), au lieu d'être seulement liée, de manière contingente, au moment où le processus s'arrête.

Pour désigner cette référence qui articule détermination quantitative et qualitative, on utilisera l'étiquette qnt/qlt : alors qu'un nominal comme eau relève a priori d'une référence qnt, un nominal comme voiture appelle une référence qnt/qlt ; alors que les verbes comme plaire et pleuvoir relèvent respectivement d'une référence qlt et d'une référence qnt, les verbes dits d'accomplissement comme réparer relèvent d'une référence qnt/qlt.

Massif versus comptable, « état» (ou propriété) versus « activité » (ou processus) 6 versus «accomplissement» : le jeu quantité/qualité se trouve par conséquent au cœur de deux des grandes oppositions référentielles, organisant respectivement le champ de la détermination nominale et celui de l'aspect verbal ${ }^{7}$. Cela lui confère un champ

6 Ces désignations sont celles que proposent Vendler ou ses traducteurs, avec l'hésitation entre état et propriété, entre activité ou processus, et aussi entre accomplissement et ce qui pour Vendler constitue la quatrième catégorie, celle des « achèvements ».

$7 \mathrm{Du}$ côté nominal, les descriptions traditionnelles ne retiennent que les deux catégories du comptable et du massif, correspondant respectivement dans nos exemples à une référence articulant détermination quantitative et qualitative pour ce qui concerne le comptable, et à une référence strictement quantitative pour ce qui concerne un massif comme eau. La tradition culiolienne cependant (voir Culioli 1999) distingue bien trois catégories, à savoir le discret, qui correspond au comptable, le dense et le compact, les deux derniers se partagent la catégorie du massif : le nominal eau dont la référence est de type quantitatif correspond à la catégorie du dense ; la catégorie du compact quant à elle est illustrée par des nominaux comme blancheur dont la référence est au contraire strictement qualitative (faisant référence à la classe de tout ce qui peut avoir cette qualité de blancheur). 
empirique déjà considérable, avec une série de critères à disposition qui sont relativement opératoires pour distinguer les trois modes de référence mis en évidence :

- du côté des nominaux, ce sont tous les critères qui dans une langue donnée permettent de distinguer comptables et massifs :

(i) En français, pour les cas de référence qnt/qlt, la possibilité d'utiliser le déterminant un ou des déterminants cardinaux (deux, trois, etc.), et la possibilité de pluraliser.

(ii) En français, pour les cas de référence qnt, la possibilité d'utiliser le partitif $d u$, diverses contraintes pour l'utilisation du déterminant un (utilisation d'un quantifieur qui vient formater la référence comme dans un verre d'eau; ou coprésence d'un adjectif comme dans une eau pure), pour l'utilisation des cardinaux, et pour l'utilisation du pluriel (contraintes moins connues : voir les eaux de Venise ou les sables du désert analysés dans Jarrega 2000).

- du côté de l'aspect, ce sont les différents critères proposés par Vendler et ses successeurs :

(i) Pour les cas de référence qlt (les états ou propriétés), l'impossibilité en français d'utiliser la locution verbale être en train de, qui supposerait un ancrage quantitatif : on connaît les contraintes sur *Il est en train de savoir ; ?? Il est en train de me plaire est lui-même un exemple intéressant, car la présence de être en train de fait bien basculer plaire du coté d'une référence plus quantitative, supposant en l'occurrence que le prédicat fasse référence à un processus en cours de développement, que ce processus soit une activité ostensiblement plaisante (ce qui serait plus facile avec déplaire) ou une montée du sentiment positif ressenti par le récepteur « je ».

(ii) Pour les cas de référence qnt (les processus ou activités), l'impossibilité en français d'utiliser l'auxiliaire aspectuel finir de (*Il finit de plewvoir), qui suppose une configuration qualitative du procès.

(iii) Pour les cas de référence qnt/qlt (les accomplissements), le contraste bien connu entre pendant et en (Il a réparé la voiture pendant 5 minutes / Il a réparé la voiture en 5 minutes), le second signifiant que la finalité du processus est atteinte, et donnant dès lors au verbe une référence qui est bien à la fois quantitative (quelque chose a eu lieu) et qualitative (la voiture est réparée) ; ou aussi le contraste entre cesser de et finir de (il a cessé de réparer la voiture / Il a fini de réparer la voiture qui veulent dire l'un que le processus s'est arrêté, l'autre qu'il a continué jusqu'au bout).

Ce qui se présentait comme une différence relativement abstraite s'avère par conséquent recouvrir des comportements empiriques parfaitement concrets et identifiables.

\section{Polysémie lexicale et grammaticale}

\subsection{Les polysémies régulières}

L'intérêt premier de cette distinction entre référence qut, référence qlt et référence qnt/qlt n'est pas de permettre le classement des unités en catégories, dans la mesure où l'on observe constamment des jeux de bascule d'une catégorie à l'autre (comme ci-dessus pour plaire) en fonction des opérations linguistiques affectant les unités en question : ainsi qu'on l'a annoncé au départ, la distinction entre ces trois 
modalités de référence permet surtout de rendre compte de faits de variation, et en l'occurrence de faits de polysémie, avec des unités qui peuvent relever des trois catégories, et voient de ce fait leur valeur interprétative varier.

On rencontre là à nouveau des faits connus, en tous les cas dans le domaine nominal, où les passages du massif au comptable et vice-versa sont bien étudiés : si on admet par exemple que chien est comptable, on rencontre néanmoins sous diverses modalités des emplois massifs (J'ai mangé du chien par exemple). L'ensemble de ces variations a précisément été décrit comme mettant en jeu une " polysémie régulière » (Nunberg \& Zaenen 1997).

Dans le domaine aspectuel, on sait moins qu'il y a aussi circulation entre les différentes catégories vendleriennes. On peut citer par exemple le verbe boire, qui peut relever d'un fonctionnement qlt (1a), autant que d'un fonctionnement qnt (1b), ou d'un fonctionnement qnt/qlt (1c):

(1a) Il a bu mais il ne boit plus.

(1b) Hier, il a bu et il a dansé.

(1c) Ca y est, il a bu.

C'est ce qui ressort des critères qui ont été rappelés ci-dessus : (1a) admet bien une interprétation dans laquelle il est question d'inscrire «il» dans la catégorie des (anciens) alcooliques, en quel cas il est fait référence à une époque dans laquelle le procès de boire n'avait pas lieu en permanence, et donc dans laquelle il n'était pas « en train de boire » en permanence ; (1b) fait référence à un événement qui est bien situé (" hier»), et qui serait compatible avec une description indiquant sa durée (« il a bu pendant de longues heures »); (1c) admet un circonstanciel de durée en en qui indique l'atteinte de la finalité du processus.

La variation en question correspond à des opérations linguistiques différentes : effet de contraste opéré par « mais non p » en (1a); localisation temporelle « hier » qui déconnecte les procès de la situation d'énonciation en (1b); ancrage situationnel marquant un changement d'état via «ça y est» en (1c). Mais elle a des effets sémantiques suffisants pour que l'on puisse invoquer un cas de polysémie : en (1a), boire prend, si on lui confère une référence de type qualitative, le sens d'être alcoolique ; en (1b), il a le sens réputé premier qui est celui renvoyant à une simple absorption de liquide ; en (1c), il prend à nouveau un sens marqué, qui peut être celui de "s'enivrer" ou simplement de "se désaltérer ", et qui correspond au fait que le processus s'est vu doté d'une mesure de complétude le configurant (la complétude en question correspondant à la finalité aristotélicienne et se mesurant sur l'état résultant de cette absorption - ivresse ou résorption de la soif).

\subsection{Généralisation : l'opposition concret / abstrait rendue à sa profusion}

Le mécanisme de variation qui vient d'être rapidement illustré est en fait extrêmement productif, et rend compte d'une part non négligeable des faits de polysémie auxquels les unités morpho-lexicales donnent lieu. En effet, toute entité référentielle peut être de type qnt, qlt ou qnt/qlt. Or le fait que les unités morpholexicales, au lieu d'être simplement des unités isolées, entrent dans des constructions, a pour conséquence que chacune se trouve inscrite dans des relations entre potentiellement 
plusieurs unités : cela leur confère un potentiel de variation qui peut dès lors être démultiplié, chacune des entités référentielles mobilisées par ces unités pouvant relever en droit d'une référence qnt, qlt ou qnt/qlt.

C'est ainsi que sur le verbe filer De Vogüé (2004) a pu distinguer tout un faisceau de valeurs différenciées pouvant être rapportées à ce jeu du quantitatif et du qualitatif. L'idée générale est que filer met nécessairement en scène :

(i) une entité « fil » au titre d'objet interne,

(ii) le référent de son sujet, occupant la fonction d'être ce qui file,

(iii) ce à quoi réfère le verbe lui-même : ce que le référent du sujet est ou fait, autrement dit le mode d'être de ce référent,

(iv) un quatrième paramètre reliant entre eux les précédents, qui est une forme de parcours dont le fil (i) est la manifestation, et qui est ce qui caractérise le mode d'être (iii) en question.

Selon que cette manifestation est plus ou moins quantitative (quelque chose se manifeste, fil ou filage) ou plus ou moins qualitative (filer est prédiqué d'une entité indépendante), on aura des valeurs où le verbe met en scène de vrais fils concrets (comme quand c'est le gruyère qui « file »), ou au moins des manifestations concrètes de filage (comme quand c'est Mesrine qui « file»), ou des valeurs où c'est seulement la propriété d'être filiforme qui se manifeste (comme quand c'est le temps qui « file»). On voit que cela correspond à des valeurs ressenties comme plus concrètes ou plus abstraites selon les cas, et qui seraient en tout état de cause classées comme telles dans des analyses fondées sur l'opposition entre valeurs concrètes et valeurs abstraites.

Il y a cependant aussi les autres entités impliquées (ii, iii, et iv), qui elles aussi peuvent être selon les cas plus ou moins quantitatives, ou plus ou moins qualitatives. Elles varient non seulement en elles-mêmes mais surtout dans leurs relations les unes avec les autres : de la même façon qu'il peut y avoir plusieurs modalités de manifestation selon que la manifestation est quantitative ou qualitative comme on vient de le voir, il peut y avoir plusieurs formes de modes d'être selon que le mode en question est une quantité (et le mode d'être est alors une simple façon contingente d'être, un alea) ou une qualité (et ce sont alors les propriétés de l'entité qui sont en jeu, voire son essence même, ce qui la fait « être ») ; et il peut y avoir plusieurs façons pour le parcours de "caractériser» ce mode d'être, selon qu'il s'agit de le caractériser quantitativement (d'en être un élément constituant, donc une partie) ou qualitativement (d'en être alors une propriété constitutive). Il n'est pas possible dans le cadre de cet article de reprendre le détail de la démonstration, mais on en arrive ainsi à distinguer toutes les formes que peut prendre le filage selon que c'est le gruyère ou le caramel qui file (le caramel devient caramel quand il file, ce qui n'est pas le cas du gruyère: pour le caramel, le mode d'être n'est pas un alea contingent mais un avènement, puisque c'est l'apparition de fils qui marque qu'on a obtenu du caramel), selon que c'est le train ou le chemin qui file (avec le chemin rien ne se passe), selon que c'est le train ou le fugitif (avec pour le fugitif la finalité qui est d'avoir fui et donc de disparaître, ce qui n'est pas le cas du train : ainsi a-t-on des emplois où « File!» signifie de disparaître, et d'autres où l'injonction signifie d'aller vite). 
Au lieu de s'en tenir à l'opposition binaire concret/abstrait, le jeu qnt/qlt peut ainsi rendre compte de toutes les variantes que recouvre le verbe (ici dans ses emplois intransitifs), selon qu'il s'agit de vitesse, de disparition, de production de fil, de simple effet de linéarité, de processus de déstructuration (le pouls qui file, et d'une autre façon le bas qui file), etc. La métaphore du fil pourrait sans doute elle aussi les recouvrir toutes, mais sans donner accès au détail des variations, et sans rendre compte des variantes quand il n'y a pas métaphore, mais bien un fil concret, qui pourtant peut intervenir de différentes façons (l'ancre file, le caramel file, le gruyère file, le bas file). Le jeu qnt/qlt ne dit pas que toute extrapolation est possible, il vise à dire ce qui est possible, et il en déploie la description détaillée. Les effets qu'il induit peuvent en outre être sensiblement différents d'une unité lexicale à l'autre, à la mesure du réseau de relations que tisse chaque unité : cela dépend du nombre des entités impliquées, de leur nature, de la nature des relations qu'elles entretiennent, et on a vu que l'opposition qnt/qlt ne se jouait pas de la même façon lorsqu'il s'agit de "manifestation", de "constitution» ou de "mode d'être ». C'est donc tout un faisceau de nuances complexes qui peuvent ici être déployées, au lieu d'une machine universelle assimilant les différences et lissant les singularités.

\subsection{Du côté des catégories grammaticales : l'exemple du présent}

Le lexique n'est pas le seul lieu où s'observent ces variations de valeurs. Les exemples (1a-c), choisis pour déployer les variations auxquelles peut être soumis le verbe boire, illustrent par la même occasion la variation à laquelle donne lieu le passé composé : ou du moins ils illustrent une partie de sa variation, celle qui se trouve être la plus connue et la mieux répertoriée, entre une valeur dite temporelle de passé en (1a) et (1b), et une valeur dite aspectuelle d'accompli dans le présent en (1c). On pourrait multiplier les exemples du côté des marqueurs grammaticaux, et en particulier des marques de flexion verbale, qui sont pour la plupart nettement polysémiques, bien que la polysémie en question tienne aussi à d'autres mécanismes que celui du jeu entre quantité et qualité qui nous occupe ici. On propose ici d'illustrer ce jeu sur le cas des énoncés dits "au présent » ${ }^{8}:$ la variation qui s'y observe permet, plus directement qu'une autre, d'introduire à une variation plus large, qui porte sur les types de discours et le positionnement énonciatif, et que l'on analysera en C.

Le champ de valeurs recouvert par ce que l'on appelle le présent est important. Il se laisse cependant lui aussi répartir en trois domaines suivant les effets du jeu entre modalités quantitatives ou qualitatives de construction de la référence\%. On illustrera ces trois domaines en se fondant sur l'ambiguité de l'exemple (2) :

(2) Il fait la vaisselle.

(2) peut avoir une interprétation générique, en quel cas il n'est pas question de dire que «il » est effectivement en train de faire la vaisselle au moment où on parle, mais simplement d'indiquer qu'il est de ceux à qui il arrive de la faire, sans que soit

8 Dans la mesure où une analyse morphologique un peu précise montre clairement qu'il n'y a pas de marque de présent en français, il n'y a pas de sens à parler d'une polysémie du présent : la variation dont il va être question est celle des structures énonciatives minimales elles-mêmes, ce qui permettra d'autant mieux la transition avec l'analyse des types de discours et celle de la syntaxe.

9 Sur le présent, voir (De Vogüé 2000). 
précisé quand et à quelle fréquence il l'a fait effectivement. On reconnaît là les différents critères qui caractérisent une référence qualitative : renvoi à une classe (les faiseurs de vaisselle) plutôt qu'à une localisation; incompatibilité avec être en train de.

(2) peut avoir aussi une interprétation descriptive, ce qui va alors correspondre à la valeur souvent donnée comme étant la valeur première ou valeur propre du présent, à savoir la valeur déictique ${ }^{10}$; le fait qu'elle soit déictique signale assez qu'il s'agit d'un cas de référence située, et donc quantitative ; et elle peut au demeurant être située à un autre moment que le moment de l'énonciation, par un effet de translation bien connu : il est de ce point de vue remarquable que cet énoncé descriptif suppose nécessairement un tel ancrage situationnel, au point de l'induire implicitement quand celui-ci n'est pas auparavant établi (contextuellement ou situationnellement), s'interprétant alors comme une description partielle d'une situation plus large que l'énoncé a suffi à introduire dans la scène référentielle :

(2b) Il fait la vaisselle. Elle lit. Au loin on entend des rires d'enfants...

Sans doute l'insertion de l'auxiliaire finir comme aussi l'utilisation du duratif en sont-elles possibles, dans la mesure où l'objet défini la vaisselle suffit à doter le procès d'une finalité (Il finit de faire la vaisselle; Il fait la vaisselle en deux minutes). Mais le propre de cette interprétation est précisément de donner une saisie du procès comme étant « en cours »: ce que l'on appelle une saisie sécante qui correspond au fait que sa finalité n'est pas atteinte encore. « Il » est en train de faire la vaisselle, et rien ne garantit qu'il la finira si d'aventure quelque événement vient l'interrompre.

C'est tout le contraire dans le cas d'une interprétation narrative de (2), interprétation correspondant à la valeur dite de présent narratif, dans laquelle le présent ne renvoie pas au moment de l'énonciation mais à un moment passé ou fictif, et dans laquelle la saisie du procès n'est plus sécante mais globale, comme ce serait le cas avec un passé simple :

(2c) Une fois qu’il a dîné, il regarde le programme de la soirée télé. Il fait la vaisselle. Puis il s'installe dans son fauteuil devant la télé.

Aussi longtemps qu'ait pu durer cette vaisselle, l'énoncé l'embrasse entièrement, jusqu'à son terme, vaisselle faite, et la donne par conséquent comme constituant un bloc insegmentable, subsumé en totalité par la finalité qui la constitue. Ce type d'interprétation, généralement présenté comme une valeur marquée, paraît en fait souvent la valeur la moins coûteuse, en particulier avec les verbes que Vendler a reconnus comme des verbes d'achèvement, pour lesquels la valeur descriptive et donc déictique est plus difficile à obtenir : sauf dans un reportage en direct (qui reste une

10 Noter qu'un énoncé effectivement déictique a en fait des conditions d'emploi assez difficiles à réunir, puisqu'il s'agit qu'il décrive ce qui est en cours et donc pourrait être constaté au moment où il est proféré. Pour qu'il y ait ainsi coïncidence entre le dire et le dit, il faut que l'on soit à parler pour dire à l'autre, présent, tout ce qui est autour de lui et qu'il voit bien («Nous sommes tous les deux dans une pièce, il y a une table, deux chaises, des assiettes, un poulet à moitié découpé, nous mangeons... »); c'est même l'une des propriétés du langage et des signes en général que d'avoir pour vocation première de faire avec l'absence. En fait, la fameuse valeur propre du présent est réservée à des cas, étranges, où la situation, opaque, se trouve diagnostiquée en direct par le locuteur ("ça y est, j'ai compris, il fait la vaisselle »), ou à des cas, cette fois fréquents, où l'auditeur ne voit pas la scène de référence (une déicticité qui serait donc temporelle, mais seulement partiellement spatiale), et où le locuteur donne donc une information (" qu'est-ce qu'il fait dans la cuisine ? - Il fait la vaisselle. ») 
narration, bien que portant sur le moment présent) ou en cas d'effet d'annonce (Attention il ouvre la porte!), un énoncé comme Il ouvre la porte s'interprète bien plus naturellement dans un cadre narratif, en relation donc à des faits passés ou fictifs (Il ouvre la porte, il la voit, il ressort), que dans un cadre descriptif, avec référence au moment d'énonciation (il y aurait là en outre un effet de ralenti nécessaire et forcément couteux : «- Qu'est-ce qu'il est en train de faire là, à cogner comme un sourd sur cette porte? - Il ouvre la porte, pourquoi?»). Cette valeur narrative relève bien de la référence mixte qnt/qlt, où c'est la finalité qui détermine la délimitation du procès : le duratif en est possible et n'a pas les mêmes conditions de vérité que le duratif pendant (vaisselle faite avec en, pas nécessairement avec pendant) ; et les auxiliaires finir et cesser s'opposent nettement, avec finir signifiant qu' «il » continue pour aller jusqu'au bout, quand cesser dit que le procès s'interrompt.

Le contraste entre ces trois modes de construction de la référence du procès se manifeste aussi au travers de l'interprétation du coordonnant $e t$, qui doit être à son tour tenu pour un marqueur polysémique dans les mêmes conditions que tous ceux que nous avons examinés ici :

(3) Il fait la vaisselle et il sourit.

Dans une interprétation générique de (3), et marque une forme de cumul de propriétés, «il » étant présenté comme doté de la double propriété d'être un faiseur de vaisselle et d'être une personne souriante; dans une interprétation descriptive, et a une valeur de concomitance, correspondant donc à l'ancrage situationnel caractéristique de cette interprétation quantitative («il » fait deux choses en même temps : la vaisselle et un sourire); dans une interprétation narrative, et prend la valeur d'une marque d'enchaînement (d'abord «il» fait la vaisselle, et ensuite, une fois qu'il a terminé, il se met à sourire), correspondant à une construction progressive du temps (et non plus seulement transitoire comme dans l'interprétation descriptive) bien étudiée dans la littérature portant sur la sémantique des temps et des aspects ${ }^{11}$.

Le contraste qui nous intéresse ici s'avère donc avoir des effets en série, sur l'interprétation des unités, mais aussi sur les valeurs aspectuelles, et encore sur l'inscription dans le temps des procès : hors temps avec la référence qlt ; pris dans un temporalité construite comme transitoire avec la référence qnt; pris dans une temporalité évolutive où les faits s'enchainnent avec la référence qnt/qlt, le temps étant alors organisé en termes de changements et de passages de bornes plutôt que d'intervalles plus ou moins stationnaires.

On va voir maintenant qu'il a aussi des effets sur l'ordonnancement général du discours.

\section{3. Énonciation et référence propositionnelle}

\subsection{Une relecture référentielle des types de discours}

Les trois valeurs du présent décrites dans la séquence précédente se sont trouvées rapportées à des activités énonciatives différentes : catégorisation d'un individu (dans

11 Voir en particulier (Dowty 1986), et pour des discussions de différents points de vue sur ces effets d'ordre temporel (Moeschler et al. 1998). 
la classe des faiseurs de vaisselle) pour la référence qlt, description pour la référence qut de (2b), narration pour la référence qnt/qlt de (2c). Ce sont là des types de discours bien répertoriés. On propose ici de les rapporter à des modalités différentes de construction de la référence. Que la catégorisation d'individus mobilise des prédicats qualitatifs est attendu ; il est moins évident en revanche que la description puisse se ramener à une affaire de référence quantitative ou que la narration soit affaire de référence quantitative délimitée qualitativement.

Pour la description, cela revient à soutenir qu'elle mobilise des prédications d'existence - il s'agit de dire ce qu'il y a -, et qu'elle est nécessairement située : ce que l'on décrit est une situation, ou une entité prise dans une situation. Sans doute les descriptions passent-elles par l'attribution de propriétés, et l'on pourrait penser qu'elles restent à ce titre des constructions qualitatives : les mots utilisés, comme toutes les unités lexicales, mobilisent bien des propriétés et du qualitatif (la qualité de chien, la qualité du sommeil, la qualité d'être en boule, la qualité d'être un panier etc., dans une description qui dirait un chien dort en boule dans un panier). Reste qu'avec ces mots au contenu qualitatif, l'activité descriptive consiste à «rendre» la scène à décrire, en reconstituant ce qui s'y trouve : il ne s'agit pas de catégoriser scènes ou entités, mais bien de poser ou de « camper »; les mots utilisés ont pour fonction non pas d'attribuer des propriétés à des éléments déjà donnés, mais d'introduire ces éléments. En outre, l'objet de la description est avant tout de rendre ce qui est perceptible dans la situation considérée. Dès lors, les propriétés mobilisées peuvent ne caractériser les éléments campés que de manière contingente et transitoire (il dort là juste, mais il ne dort pas toujours); et, même lorsqu'il s'agit de propriétés essentielles, elles ne sont mobilisées que pour mieux identifier ces éléments dans la situation concernée (celui-là est un chien qui dort, et à coté, il y a un chat qui est noir et qui ronronne, et à coté encore un arbre, en fleurs, etc.). C’est donc le mode de présence de ces éléments dans la situation décrite qui est en jeu, plutôt que ce qui pourrait constituer leur essence propre. A partir du moment où l'on veut bien entendre la différence qu'il peut y avoir entre ce qui est en jeu dans une simple description et ce qui procède d'une catégorisation, à partir du moment donc où l'on veut bien ne pas appeler description tout ce qui n'est pas dynamique, on doit admettre qu'il puisse y avoir d'autres lignes de partage que celle qui oppose staticité et dynamicité : la distinction majeure entre le thétique et le catégorique d'abord, et celle qui s'avère liée entre modes de présence transitoires et propriétés essentielles ensuite, qui renvoie non seulement à des types de prédicats différents, mais plus fondamentalement à des types de prédication différenciés.

Cette distinction entre deux types de prédicats est au demeurant bien connue de la sémantique référentielle contemporaine, et on va voir qu'elle se rapporte effectivement à une opposition entre quantité et qualité. C'est au départ Carlson (1977) qui a proposé de distinguer entre ce qu'il appelle les prédicats d'individu (i-predicates) et ce qu'il appelle les prédicats « de stage » (s-predicates), les seconds étant donnés comme transitoires, et caractérisant non pas l'individu, mais un «stage » - un état - de cet individu. Cette distinction s'impose dès lors que l'on prend au sérieux la notion de prédicat catégorisant : on ne voit pas en quoi le fait qu'il soit à un moment donné en train de dormir, d'être assis, d'être près d'un arbre, ou d'être épuisé pourrait catégoriser un individu; cela ne fait que catégoriser l'état ou la situation dans lesquels il se trouve. Mais cette caractérisation transitoire s'avère en outre être nécessairement associée à 
une prédication d'existence dans une situation particulière, et donc à cette modalité quantitative de construction de la référence à laquelle nous prétendons rapporter la description. Le contraste entre i-prédicats et s-prédicats a en effet été mis en évidence au départ pour rendre compte des conditions de prédication sur un sujet indéfini : (4) ci-dessous est nettement plus contraint que (5), tandis que (6), avec un prédicat clairement transitoire et une localisation situationnelle (dans un coin), est parfaitement construit :

(4) ?? Un homme est médecin ${ }^{12}$.

(5) Pierre est médecin.

(6) Dans un coin, un homme est allongé.

Or, par opposition aux définis, les déterminants indéfinis ressortissent bien à une prédication d'existence : le groupe nominal pose qu'il y a l'homme dont il est question, au lieu de simplement référer à un homme indépendamment défini. Le contraste (4)-(6) montre que cette prédication d'existence est incompatible avec des prédicats catégorisants et appelle des s-prédicats. Il s'avère en outre que l'ensemble sera d'autant mieux construit qu'il est lié à un ancrage situationnel (Dans un coin) $)^{13}$. Et il s'avère enfin que l'énoncé s'inscrira ce faisant dans une démarche descriptive. L'ensemble de ces contraintes s'explique dès lors que l'on a pris la mesure de ce qu'est une description selon la caractérisation qui vient d'en être donnée : un travail énonciatif consistant à rendre une situation (d'où le caractère à la fois localisé, transitoire de toute description) par la prédication d'existence d'entités qui la constituent (qui la constituent partiellement, d'où le caractère non exhaustif des descriptions), dont on s'efforce de restituer le mode de présence (s-prédicats) dans la situation en question.

Quant à la narration, elle se laisse caractériser sans doute par le dynamisme des événements qu'elle rapporte, mais d'abord par le fait même qu'il s'agisse d'événements. En effet la notion d'événement renvoie à cette délimitation télique des faits, quand ils se trouvent associés à un changement qualitatif qui leur donne leur finalité : un événement est un procès de type qnt/qlt. Or cette délimitation télique est ce qui confère à l'enchaînement des faits son caractère dynamique : la fin du procès marquant un changement qualitatif entre un avant et un après ; elle n'est plus un simple arrêt, mais bel et bien un accomplissement. Avant même donc que de parler du mode de structuration du texte narratif, ce qui définit ce type de discours est la référence qnt/qlt, qui fait des procès des événements.

Notons que le caractère particulier des événements comme types de procès est lui aussi bien connu de la littérature linguistique. Il est évidemment connu des théories de l'aspect. Mais il l'est aussi des théories qui constituent le champ de la sémantique référentielle, depuis qu'il a été montré par Davidson (1967) que certaines propositions référaient à des événements, les divers prédicats constituant la proposition portant sur ce référent événement et non sur le référent des groupes nominaux impliqués dans la proposition : dans (7) ci-dessous, le groupe verbal et aussi les différents compléments

12 Parmi les conditions pour rendre (4) énonçable, il y a la possibilité d'en faire un énoncé générique (plus accessible avec des exemples classiques comme Un soldat salue devant le drapeau; ici il faudrait que l'énoncé signifie que tout homme est et doit être médecin, ce qui constitue une interprétation un peu difficile à reconstituer).

13 Voir Dobrovie-Sorin (1997). 
circonstanciels s'entendent comme des prédicats qui se trouvent appliqués non pas au référent du sujet, mais à l'événement e que l'énoncé relate :

(7) Pierre a violemment frappé Jean dans le dos avec un couteau.

Le fait de «frapper» caractérise d'abord ce que Pierre a fait, même s'il est possible d'en inférer éventuellement une caractérisation de Pierre ; c'est l'acte qui est donné comme étant violent; c'est lui, et non pas Pierre ou Jean, qui se voit rapporté à la localisation «dans le dos » et se trouve qualifié comme ayant nécessité un couteau.

En introduisant cet élément " événement » dans des systèmes référentiels qui ne comportaient que des individus, des variables individuelles et des prédicats portant sur ces individus, Davidson modifiait de manière importante les contraintes portant sur ces systèmes. À sa suite, il est des sémanticiens qui ont simplement ajouté ces éléments nouveaux à l'ontologie de leur modèle, supposant que toute proposition porte à la fois sur des individus et sur des événements. Reste que la proposition de Davidson porte sur des énoncés qu'il a caractérisés comme étant des énoncés d'action : rien n'oblige à l'étendre à des énoncés de jugement comme (5); rien n'oblige à l'étendre même à des énoncés descriptifs comme (6), qui portent si notre analyse est correcte non pas sur un événement, mais sur la situation désignée par «dans un coin». Prendre acte de la variation dans ce domaine des types de discours conduit à envisager que le format référentiel des propositions ne soit pas unique et qu'il puisse varier d'un type à l'autre : seuls les énoncés narratifs sont constitués de propositions qui réfèrent à des événements, tandis que les propositions constituant les énoncés descriptifs réfèrent à des situations, et que les propositions des énoncés catégorisants réfèrent quant à elles aux individus qu'elles catégorisent. Une variation qui concerne aussi le statut du groupe verbal qui occupe la position de prédicat dans ces propositions : l'analyse de Davidson montre qu'il désigne directement l'événement en question dans les énoncés narratifs, quand il fait fonction de i-prédicat attribué au sujet dans les énoncés catégorisants, et de s-prédicat caractérisant le mode d'être des objets concernés dans les énoncés descriptifs. C'est dire que dans les énoncés narratifs, le groupe verbal a directement une fonction référentielle : d'une certaine façon on peut dire que c'est lui qui est le pivot de la référence de la proposition; c'est lui en tous les cas qui est l'élément référent principal, à partir duquel les autres éléments s'organisent (par exemple pour faire fonction d'actants). Dans les énoncés catégorisants et descriptifs, le prédicat porte sur le référent mais n'est pas lui-même référent: c'est le sujet sur lequel porte la prédication qui est l'élément référent dans les énoncés catégorisants; et dans les descriptifs, ce sont en définitive les compléments circonstanciels de temps et lieu, quand ils sont explicites ( $\mathrm{cf}$ «dans un coin»), qui font fonction d'élément référent principal (ils désignent la situation à laquelle l'énoncé réfère), les groupes nominaux se contentant de camper leur référence dans le cadre global que ces circonstants déterminent.

\subsection{Frege, Sémantique des Situations ou DRT (Théorie des Représentations du Discours)}

On soutient par conséquent que la structure référentielle est totalement différente selon le type de discours, avec des fonctions prédicatives qui sont différentes, avec une 
répartition de la référence sur la proposition qui est différente, avec des groupes verbaux et nominaux qui n'ont pas le même statut référentiel d'un type de discours à l'autre (puisque les groupes nominaux ne désignent le référent que dans le cas des énoncés catégorisants). Au travers de ces variations, c'est la conception même de ce qui peut constituer la référence des propositions qui se trouve engagée : derrière chacun des types de discours qui viennent d'être décrits, c'est chaque fois un nouveau modèle de la référence propositionnelle qui se trouve profilé.

Les énoncés catégorisants s'intègrent parfaitement dans un modèle de type frégéen, avec un i-prédicat qui attribue telle ou telle catégorie au référent du sujet sur lequel il porte: un modèle dont on a vu qu'il s'applique mal aux s-prédicats des énoncés descriptifs, et que l'analyse de Davidson remet en cause dans le cas des énoncés relatant ce qu'il appelle des actions. En revanche, pour les énoncés descriptifs, la Sémantique des Situations (Barwise et Perry 1983) parait mieux adaptée quand elle donne pour référents aux propositions des situations, celles-ci étant conçues comme des ensembles constitués des individus dont la proposition parle, des propriétés qu'elle leur confère, et des relations qu'elle établit entre eux. Or, ce modèle, donné comme une alternative au modèle frégéen jugé contre-intuitif parce qu'il conduit à ce que la proposition globale n'ait d'autre référent que la vérité ou la fausseté de la prédication effectuée, est lui-même largement contre-intuitif quand on l'applique aux énoncés catégorisants comme (5), dans lesquels on voit mal de quelle situation il pourrait être question, surtout si la situation est figurée comme un ensemble dans lequel le référent du sujet et la propriété qui lui est attribuée constituent deux éléments distincts. On prétend l'appliquer à toute proposition alors qu'il n'est adapté ni pour les cas où les propriétés en jeu sont des propriétés essentielles, ni pour les cas où les groupes verbaux réfèrent non pas à des propriétés ou des relations, mais à ces changements de propriétés ou de relations que sont les événements. Il permet de capter à la fois le fait que la référence soit transitoire et donc située, et le fait que toute la proposition participe à la description de la situation en question. Il n'est pas adapté pour ce qui n'est pas transitoire, mais qui est soit essentiel soit dynamique ; et il n'est pas adapté pour une référence qui au lieu de se répartir sur toute la proposition, se trouve soit organisée autour du groupe nominal sujet (ce qui est le cas des énoncés catégorisants, dans lesquels le groupe verbal n'est pas référentiel mais prédicatif), soit autour du groupe verbal (ce qui est le cas des énoncés narratifs). Plutôt que de se penser comme des modèles concurrents, le modèle frégéen et celui de la Sémantique des Situations devraient donc être donnés comme complémentaires, l'un et l'autre se partageant une partie du champ de la référence propositionnelle.

Reste qu'aucun de ces deux modèles ne paraît convenir pour rendre compte de la sémantique des énoncés narratifs et de leur dynamisme propre. Pour ceux-là, serait plus adaptée l'intuition qui est celle de la DRT de (Kamp et Reyle 1993), quand elle modélise la façon dont chaque proposition va pouvoir introduire des éléments nouveaux, et les changements qui en résultent dans l'information apportée par le discours en cours. La DRT cependant prétend aussi non seulement rassembler tous les types de discours, mais traiter uniformément tous les éléments référentiels de la proposition, groupes nominaux, groupe verbal, circonstants, quand on a vu que la référence n'est pas répartie uniformément d'un type de discours à l'autre. 
Contre ces approches uniformisantes, qui nivellent les différences ou donnent la priorité à une option par rapport aux autres, le jeu entre quantité et qualité permet de penser la variation comme une nécessité structurant le langage.

\subsection{Des structures énonciatives différenciées}

On a décrit des types de discours sans vraiment recourir à ce que Benveniste a désigné comme étant l'appareil formel de l'énonciation, mobilisant sujets, ancrages subjectifs et ancrages situationnels. C'est que, aussi importante que soit la problématique de la prise en charge, ce n'est pas sur ce plan que se distinguent d'abord catégorisation, description et narration ${ }^{14}$, mais sur celui des modalités de constitution de la référence. De ce point de vue, la dimension énonciative ne saurait être rejetée dans un après de la sémantique, une fois la référence constituée : structuration discursive et structuration référentielle ne peuvent pas être séparées, l'énonciation se joue au cœur de la sémantique. De fait, il n'y a aucune raison que l'énonciation se réduise à la façon dont un sujet énonciateur énonce et s'énonce : l'énonciation recouvre le procès de constitution d'un énoncé, procès dans lequel il n'est pas forcément crucial qu'un agent s'implique, mais dans lequel en revanche il faut bien que quelque chose s'énonce, ce qui suppose d'abord la structuration d'une référence pour cet énoncé ${ }^{15}$.

En distinguant trois grands types de référence, on montre aussi que l'énonciation ne passe pas toujours par des procédures de repérage situationnel. Des trois, seule la description a été rapportée à un ancrage situationnel. Dans les deux autres types, il n'y a pas de situation en cause, et en particulier la question de la relation qui pourrait se mettre en place entre la référence de l'énoncé et la situation d'énonciation ne se pose pas. C'est ce que les observations sur les énoncés au présent ont mis en évidence : dans les énoncés catégorisants, la référence est hors temps et il n'y aurait aucun sens à la rapporter de quelque façon au moment de l'énonciation ; dans les énoncés narratifs, la référence est décrochée du moment de l'énonciation, dans un passé séparé, ou dans une simple fiction; seuls les énoncés descriptifs sont déictiques, ou éventuellement rapportés à une situation autre dans laquelle l'énonciation se translate.

L'observation des jeux qui s'instaurent entre référence quantitative et qualitative incite par conséquent là encore à refuser toute approche uniformisante, et à considérer qu'il y a plusieurs modèles de l'énonciation, celui des descriptions qui mobilisent crucialement du repérage situationnel (parce qu'il s'agit de dire ce qu'il y a, et qu'il faut alors situer cet il y a), les autres qui ressortissent à d'autres mécanismes : les catégorisations mobilisent un repérage d'un tout autre ordre, qui est notionnel (parce qu'il s'agit de rapporter le référent du sujet à une notion et à la catégorie que cette notion subsume); les narrations relèvent de repérages qui sont relatifs à l'enchaînement

14 Encore que la prise en charge soit l'un des éléments qui distinguent les trois types : garant de la vérité dans le cas de la catégorisation, qui oppose le vrai au faux; point de vue observateur dans le cas de la description, qui organise le parcours suivi par cette description; point de vue des personnages dans la narration, où l'effet de rupture correspond au fait que l'on se trouve transporté dans l'univers narré, et donc extrait de son propre univers, pour adhérer aux personnages et les suivre.

15 Voir De Vogué (1992) où l'on soutient que cette conception large de l'énonciation est l'un des points qui caractérisent la théorie du langage développée par Antoine Culioli, par opposition à des conceptions plus étroites liant l'énonciation à l'expression d'un énonciateur. 
des événements les uns par rapport aux autres, ainsi qu'illustre l'exemple (2c) ou aussi l'exemple (3) dans son interprétation narrative, cet enchaînement venant en quelque sorte se substituer à une prise en charge subjective pour organiser le récit ${ }^{16}$.

Sans doute trouve-t-on dans la littérature des typologies variées, qui varient entre autres par le nombre de types distingués, et qui peuvent en outre se surajouter les unes aux autres, les unes étant plus relatives au positionnement énonciatif, les autres à la structuration du texte, sans compter les différenciations relatives au genre. Il n'est pas question de fait de réduire à trois les types de discours possibles (comme il n'a pas été question de réduire à trois les valeurs du présent ou celles de n'importe quel lexème). On soutient seulement que l'un des paramètres cruciaux de différenciation est relatif aux modalités de la référence quantitative et / ou qualitative. Mais on s'oppose ce faisant à l'idée qu'il faudrait rapporter systématiquement tous les énoncés à la situation d'énonciation, et que la structure énonciative se mesurerait à ce rapport. Certains énoncés s'organisent sur un tel repérage, d'autres s'organisent autrement.

\section{Des syntaxes}

Les trois types de discours distingués engagent comme on l'a vu une répartition différente de la référence sur les éléments constituants de la proposition, avec en particulier pour les énoncés descriptifs une distribution uniforme, dans un cadre éventuellement implicite fourni par les circonstants, avec pour les énoncés catégorisants un ancrage référentiel centré sur le groupe nominal sujet, et pour les énoncés narratifs un ancrage centré sur le groupe verbal. C'est que la structure même des propositions se trouve affectée par ce mode de constitution de la référence, avec à chaque fois un canevas syntaxique différencié.

\subsection{Propositions bipartites, tripartites ou pluripartites}

La référence qualitative, en tant qu'elle ressortit à une prédication catégorique, met en jeu une syntaxe bipartite, avec un groupe verbal qui sert globalement de prédicat, portant sur le groupe nominal sujet :

\section{(8) Prop. $=$ GN-GV}

C'est ce modèle qui a été l'un des fondements de la conception de la grammaire développée par Chomsky, contre une grammaire traditionnelle qui était tripartite, avec sujet, verbe et objet, et en référence à un héritage logique qui s'articule sur l'opposition entre prédicats et arguments. Pourtant une telle structure prédicative paraît bien mal adaptée pour des propositions narratives, dans lesquelles le groupe verbal ne fait pas figure de prédicat, et dans lesquelles il $\mathrm{y}$ a un sens en revanche à distinguer un objet comme visée du procès auquel réfère le verbe, cette visée étant alors ce qui définit la finalité qui sert de délimitation qualitative au procès : l'objet peut sans doute avoir d'autres fonctions dans la structuration référentielle de l'énoncé, mais l'une de ses fonctions est bien d'être la mesure de la réalisation du procès, mesure qui s'évalue au

16 On pense ici à la célèbre formule de Benveniste (1966, p. 241): «Personne ne parle ici ; les événements semblent se raconter eux-mêmes (...) à mesure qu'ils apparaissent à l'horizon de l'histoire ». 
fait que son référent se trouve au terme de ce procès dans un état résultant conforme à ce qui constitue la finalité de celui-ci. De fait le principe de la structure tripartite :

$$
\text { (9) Prop. = S-V-O }
$$

n’a cessé de se maintenir malgré les arguments qui avaient été développés pour montrer l'appartenance de l'objet à un groupe verbal. Il reçoit des arguments forts dans le modèle actanciel de Tesnières, à peu près contemporain des premiers travaux de Chomsky, et qui inaugure une lignée non traditionnaliste de syntaxes sans groupe verbal ; il est défendu dans différents modèles les plus contemporains, dont le modèle Sens-Texte, et dont aussi les différentes grammaires type HPSG qui concurrencent le modèle chomskien dans le paysage post-générativiste. Plutôt que de mettre en concurrence ces deux modes d'organisation de la syntaxe propositionnelle, on propose ici l'hypothèse selon laquelle les propositions ne ressortiraient pas au même mode d'organisation selon le type de référence (et donc de discours) dont elles relèvent.

Quant aux énoncés descriptifs, dans lesquels il ne s'agit pas d'attribuer des propriétés à des arguments, ni non plus de reconstituer des procès dans leur finalité, mais de camper ce qui est, dans tel ou tel cadre, on peut s'interroger sur le sens qu'il y aurait à vouloir absolument les faire entrer dans des schémas syntaxiques organisés sur le principe de la prédication catégorielle ou de la transitivité actancielle. Or, il est des structures syntaxiques qui n'entrent pas dans ces schémas : sur le plan empirique on pense aux phrases impersonnelles (Il plent des cordes) dans lesquelles il est pour le moins difficile d'isoler un sujet; on pense aussi aux phrases à éléments détachés, postposés ou antéposés, possiblement multiples, dont la fonction est bien de définir des cadres ou de camper des entités référentielles, selon une logique d'ordonnancement fondée sur l'inclusion et non plus sur les relations actancielles, avec un noyau verbal réduit d'autant, dans lequel il peut devenir difficile de reconstituer un ordre bi- ou tripartite :

(10) Paul, à Paris, son vélo, le guidon, il l'a cassé ${ }^{17}$.

Ce type de structure est ce que décrit un modèle comme celui de l'Approche Pronominale (Blanche-Benveniste et al. 1984), qui oppose la syntaxe du noyau verbal, et la macro-syntaxe des éléments détachés, et propose une analyse du noyau verbal dans laquelle les pronoms y apparaissant ne doivent pas être interprétés comme des équivalents de groupes nominaux pleins (d'où la possibilité de ces datifs que l'on dit éthiques : Je vais te lui donner), mais plutôt comme des marques de repérage par rapport à une situation qui est soit la situation d'énonciation, soit celle que définissent les éléments détachés, soit celle qui est déterminée par le contexte de manière plus ou moins explicite. Si les pronoms clitiques qui peuvent entourer un noyau verbal ne sont pas des groupes nominaux, la structure du noyau verbal n'est pas bi- ou tripartite (on ne peut dès lors ramener un énoncé comme (10) à une structure tripartite Paul a cassé le guidon de son vélo à partir de laquelle (10) serait dérivé) mais plutôt monopartite. En même temps les éléments détachés confèrent à l'ensemble une structure qui elle est pluripartite. On est donc bien dans un autre canevas syntaxique, que l'on peut noter, au moins en première approximation :

$$
\text { (11) Prop. }=(\mathrm{Sit})(\mathrm{GNa})(\mathrm{GNb})(\mathrm{GNc}) \text { Noyau-Verbal }
$$

17 Adapté d'un exemple emblématique de Culioli. 
Là encore, plutôt que de chercher à appliquer un modèle de ce type à tous les énoncés, il serait préférable de donner acte à la variation des modes de référence, en réservant les structures qui ordonnent ce modèle aux énoncés qui se trouvent effectivement susceptibles de s'intégrer dans de telles structures pluripartites, qui sont les énoncés descriptifs à référence quantitative, avec prédication thétique, ancrage situationnel, et répartition uniforme de la référence sur chaque constituant de la proposition.

La prise en compte des effets à la fois référentiels, énonciatifs et syntaxiques du jeu entre quantité et qualité conduit donc à proposer de mettre à égalité les modèles qui se disputent le terrain de la syntaxe : les différents modèles en lice sont corrects, la proposition ne possède pas un seul format syntaxique, mais trois, selon le mode de référence ${ }^{18}$.

\subsection{Les constituants de la proposition soumis à des forces divergentes}

Reste que ces trois formats qui coexistent se partagent le même espace, celui de la proposition, qui doit pouvoir servir aux trois. On comprend que cela induise des effets de brouillage, les mêmes moyens se trouvant mobilisés pour des fonctions divergentes: d'où ce verbe qui d'un certain point de vue fait groupe avec ses compléments, d'un autre se trouve isolé comme opérateur, et placé ce faisant dans une relation de transmission entre les autres éléments de la proposition, d'un autre enfin sert de tête à la proposition qu'il organise globalement; d'où cette position objet qui parfois est associée à l'état résultant comme en (12) et s'inscrit dans une structure SVO clairement transitive, parfois s'en tient à la fonction de complément verbal servant seulement à spécifier le procès décrit par le verbe comme en (13) :

(12) Il coupe le poulet.

(13) Il coupe du bois.

d'où les fonctions thématiques variables associées à la position sujet; d'où aussi l'impossibilité d'attacher une fonction unique à la position détachée, qui peut à la fois servir à identifier le thème d'une proposition prédicative, le cadre d'une proposition descriptive, ou un élément nouveau et déclencheur dans une proposition narrative :

(14a) A Paris, il dort ; ici il n’a pas le temps. (présent générique : Paris est l'argument thématique)

(14b) A Paris, il habite un deux pièces. (présent déictique : Paris est le cadre)

(14c) A Paris, il rencontre un ami. (présent narratif : A Paris s'interprète comme « une fois à Paris », ce qui manifeste le fait qu'il sert à introduire un élément nouveau)

Quand on ne l'ignore pas pour mettre en avant l'une ou l'autre des fonctions et inscrire la proposition dans un schéma unique, on décrit généralement cette plurifonctionnalité en termes de gradient, avec une hiérarchie et des fonctions plus

18 Cela ne veut pas dire qu'il n'y ait pas un nombre indéfini de constructions syntaxiques propres à structurer les propositions : d'une part il y a d'autres points de différenciation que celui-là, d'autre part, comme pour la sémantique, l’opposition quantité / qualité peut se rejouer plusieurs fois, en différents points de la proposition. Simplement au lieu de lister les constructions, on propose de les rapporter à des principes de diversification, comme celui-ci, et ainsi de les calculer. Et on propose ce faisant de penser la syntaxe comme une dynamique, avec un nombre de constructions non limité, et des constructions différenciables indéfiniment. Un calcul plutôt qu'une liste. 
Sarab de Vogüé

prototypiques que d'autres. Plutôt que d'y voir une gradation, on propose de rapporter

la plurifonctionnalité en question aux forces divergentes qu'induit le jeu quantité/ qualité entre trois types d'ordonnancement de la proposition. 


\subsection{Nombre-genre, cas, personne : des morphologies divergentes}

Travaillant sur un même espace, ces trois structures trouvent cependant dans la morphologie des modalités de marquage différenciées. Ainsi le duo constitué par le nombre et le genre se trouve-t-il affecté au sein de la proposition à l'accord entre sujet et verbe, ou plus largement entre le sujet et le syntagme attributif, syntagme qui incarne au plus près l'alignement de la proposition sur le format d'une prédication catégorique :

(15) Ces tasses sont jolies mais abîmées.

En revanche, le cas, dans lequel le verbe joue le rôle de tête au niveau de la proposition, organise les relations au verbe du sujet et de l'objet, dans une distribution qui est bien identifiée comme étant une distribution actancielle : c'est donc bien la structure tripartite SVO qui est en jeu.

Et la personne, dont la fonction est de marquer le repérage de la proposition par rapport à la situation d'énonciation, ressortit quant à elle à la structure qui a été reconnue comme celle de la référence quantitative.

Accord, rection, repérage : on a affaire à trois logiques morphologiques distinctes, que l'analyse morphologique aurait tort de confondre. Sans doute les trois types de marquages paraissent-ils confondus dans ce qui est aligné dans les paradigmes des conjugaisons verbales: c'est parce que sur les terminaisons verbales du français contemporain, personne, nombre et cas (sujet) vont ensemble. Mais ils se séparent aussi quand les pronoms de première et deuxième personne n'ont pas de genre ou de nombre (voir la démonstration de Benveniste) ou quand les groupes nominaux non pronominaux n'ont ni personne ni cas.

Prendre en compte le fait qu'ils ressortissent à des logiques référentielles et syntaxiques divergentes permet de mieux comprendre de manière générale les discordances d'alignement qui s'observent par exemple quand les pronoms personnels n'obéissant pas aux mêmes règles casuelles ou aux même règles d'accord que les groupes nominaux «pleins ». Cela permet y compris de mieux entendre les effets de convergence qui se manifestent au sein des langues : par exemple quand le cas se met à incorporer des notions de repérage, avec effets de distance, opposant proximité et distance, et interfère alors avec les prépositions. Et cela permet aussi de donner sens aux oppositions typologiques entre langues qui paraissent organiser leur syntaxe les unes sur les relations actancielles, les autres sur des relations thématiques, d'autres sur des relations de localisation personnelles (voir Kibrik 1997), même s'il faut sans doute considérer de telles oppositions comme étant trop grossières, dans la mesure où toute langue est soumise aux trois logiques, et les organise comme elle peut, avec des effets de priorité qui dépendent surtout du point de vue adopté : comme le français, toute langue est selon le type de fait considéré, d'un certain point de vue pris dans une syntaxe bipartite, d'un autre tripartite, d'un troisième multipartite ; elle l'est aussi plus ou moins selon les registres et selon les genres (voir par exemple à l'oral et dans un français plus dialogué une organisation essentiellement multipartite) ; et ainsi elle peut s'adapter à la fois à une référence qualitative, à une référence quantitative, ou à une référence qn/qlt. 
Ces logiques divergentes fournissent surtout une piste d'explication prometteuse pour rendre compte des variations diachroniques, qui pour certaines d'entre elles pourraient résulter des déséquilibres induits par les effets conjugués de l'accord, de la rection et du repérage. On pense bien sûr au fait que dans telle langue le marquage casuel disparaisse au profit de l'ordre des constituants (qui sépare le groupe nominal et le groupe verbal), ou au fait que le marquage personnel disparaisse sur le verbe pour réapparaittre sur les clitiques. Ou aux diverses analyses à la Kurylowicz selon lesquelles les langues seraient prises dans des pentes qui redéictisent leurs modalités de marquage (ligne d'explication utilisée pour décrire les évolutions des expressions du passé et du futur, avec passé composé ou futur périphrastique ; mais aussi reprise pour l'évolution des pronoms par exemple, quand ille déictique va servir à construire de l'anaphorique, etc.) : à ce type d'analyse d'un jeu à deux entre déictique et non-déictique, on opposerait plutôt un jeu à trois, avec le déictique et le narratif qui s'opposent mutuellement au troisième larron qui est le thématique (ainsi du passé composé qui sert d'abord, avec son participe proche d'un adjectif, à donner des propriétés aux actants). De manière plus générale, le fait que le même espace syntaxique se trouve aux prises avec le jeu induit par les effets divergents des modalités quantitative et qualitative de constitution de la référence explique que cet espace soit le lieu de renversements indéfinis, renversements qu'il reste à analyser dans cette optique.

\section{Conclusion}

Que ce soit sur le plan sémantique de la polysémie lexicale ou grammaticale, sur le plan énonciatif des structures discursives, ou sur le plan syntaxique des formats propositionnels, on a mis en évidence une variation systématique se rapportant au mode de constitution de la référence. On s'oppose ce faisant non seulement à des conceptions monolithiques du sens, du discours, de la syntaxe, mais aussi à des approches qui penseraient pouvoir se contenter de recenser les valeurs des unités, les types de discours, ou les formes syntaxiques : valeurs, types et formes se rapportent à des principes de diversification, qui d'une part sont généraux et élémentaires, et d'autre part sont susceptibles de se démultiplier indéfiniment, ainsi qu'on l'a vu à propos des valeurs que prend le mot fil. Il faut donc concevoir que la liste n'en soit pas close. Et surtout, il faut entendre que chacun soit le résultat d'une construction.

On a par conséquent affaire à une grammaire qui est en construction, mais en prenant la notion de construction non pas tant dans le sens particulier qui est celui des grammaires de construction ${ }^{19}$ que dans celui plus processuel qui est celui qu'à la suite de Culioli, élabore Franckel (2002), appelant à une approche constructiviste pour reconstituer les modalités de calcul du sens, de l'énonciation, et de l'agencement des unités entre elles.

$19 \mathrm{Il}$ ne s'agit pas de répertorier les constructions, ni non plus de les considérer comme des unités complexes : on serait plutôt enclin à rapporter chacune de celles qui sont répertoriées et analysées dans les grammaires de construction à des mécanismes généraux qui les constituent, dont celui de la référence qnt ou qlt; on serait donc plutôt enclin à les déconstruire. 
Effets sémantiques, syntaxiques et énonciatifs du jeu entre quantité et qualité

\section{Bibliographie}

Barwise, J., Perry, J., 1983, Situations and Attitudes, Cambridge, MIT-Bradford.

Benveniste, E., 1966, «Structures des relations de personne dans le verbe » in Problèmes de linguistique générale, 1, Gallimard, p. 225-236.

Benveniste, E., 1966, «Les relations de temps dans le verbe français » in Problèmes de linguistique générale, 1, Gallimard, p. 237-250.

Blanche-Benveniste, Cl., Deulofeu, J., Stéfanini, J., Van der Eynde, K., 1984, Pronom et syntaxe. L'approche pronominale et son application au français, Paris, Société d'études linguistiques et anthropologiques de France.

Carlson, G., 1977, «A Unified Analysis of the English Bare Plural », Linguistics and Philosophy, 1, p. 413-457.

Culioli, A., 1999, "Valeurs aspectuelles et opérations énonciatives : l'aoristique » in Pour une linguistique de l'énonciation, 2, Paris, Ophrys, p. 127-143.

Culioli, A., 1990, « La linguistique de l'empirique au formel » in Pour une linguistique de l'énonciation, 1, Paris, Ophrys, p. 9-46.

Culioli, A., 1999, «Structuration d'une notion et typologie lexicale. A propos de la distinction dense, discret, compact » in Pour une linguistique de l'énonciation, 3, Paris, Ophrys, p. 9-16.

Davidson, D., 1967, « The Logical Form of Action Sentences » in Essays on Actions and Events, 1980, Clarendon Press, p. 105-121.

DE VogüÉ, S., 1992, «Culioli après Benveniste : énonciation, langage, intégration », LINX, 26, Université Paris Ouest Nanterre La Défense, p. 79-108.

De VogüÉ, S., 1999, "Construction d'une valeur référentielle : entités, qualités, figures », Travaux linguistiques du Cerlico, 12, Presses Universitaires de Rennes, p. 77-106.

DE VOGÜÉ, S., 2000, « Calcul des valeurs d'un énoncé 'au présent' », Travaux de linguistique, 40, Bruxelles, Duculot, p. 31-54.

De Vogüé, S., 2004, «Syntaxe, référence et identité du verbe filer», LINX, 50, p. 135-168.

Dobrovie-Sorin, C., 1997, «Classes de prédicats, distribution des indéfinis et la distinction thétique-catégorique », Le gré des langues, 12, Paris, L’Harmattan, p. 58-97.

DowTy, D., 1986, "The effects of aspectual class on the temporal structure of discourse : semantics or pragmatics?", Linguistics and Philosophy, 9, p. 37-61.

FRANCKEL, J.-J., 2002, «Introduction : Le lexique, entre identité et variation », Langue française, 133 , p. 3-15.

Jarrega, M., 2000, Le rôle du pluriel dans la construction du sens des syntagmes nominaux en français contemporain, Thèse de Doctorat, Université Paris X Nanterre.

Kamp, H., ReYle U., 1993, From Discourse to Logic. Introduction to Modeltheoretic Semantics of Natural Language, Formal Logic and Discourse Representation Theory, Kluwer. 
Sarah de Vogüé

KIBRIK, A., 1997, «Beyond subject and object : Toward a comprehensive relational typology », Linguistic Typology 1, p. 279-346.

Kratzer, A., 1995, «Stage-level and individual-level predicates », in Carlson, G., Pelletier, F. (eds), The Generic Book, University of Chicago Press, p. 125-175.

Kuroda, S.-Y., 1973, «Le jugement catégorique et le jugement thétique : exemples tirés de la syntaxe japonaise », Langages, 30, p. 81-110.

Kurylowicz, J., 1965, « The evolution of grammatical categories », Diogène, 51, p. 51-71.

Moeschler, J. et al., 1998, Le temps des événements, Editions Kimé.

Nunberg, G., Zaenen, A., 1997, «La polysémie systématique dans la description lexicale », Langue française, 113, p. 12-23.

Vendler, Z., 1967, «Verbs and Times », in Linguistics and Philosophy, Ithaca, Cornel University Press, p. 97-121. 
Effets sémantiques, syntaxiques et énonciatifs du jeu entre quantité et qualité 